\title{
基于伪频响函数矩阵法的运行模态分析方法 ${ }^{*}$
}

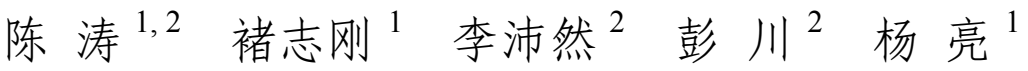 \\ (1. 重庆大学机械与运载工程学院 重庆 400044;
}

2. 中国汽车工程研究院股份有限公司 重庆 401122)

\begin{abstract}
摘要: 基于传递率测量的伪频响函数法运行模态分析技术无需白噪声激励假设, 具有强 “谐波模态抑制” 能力, 但其模态识 别结果依赖待分析伪频响函数的选取、稳定性欠佳。为解决该问题，建立了基于伪频响函数矩阵法的运行模态分析方法。该 方法首先构造多参考测点、多参考工况的伪频响函数矩阵, 然后基于伪频响函数矩阵采用最小二乘复频域法求解模态频率和 阻尼比、基于传递率函数矩阵求解模态振型, 最后通过建立相应的稳态图并结合以多参考测点平均的传递率函数矩阵第 2 阶 奇异值的倒数为基础的模态指示函数来确定模态阶数与最终的模态结果。5 自由度质量-弹簧-阻尼系统仿真结果及自由梁的运 行模态试验结果均表明，提出方法既继承了基于伪频响函数法的运行模态分析方法的强 “谐波模态抑制” 能力，亦能有效解 决伪频响函数法识别结果稳定性欠佳的问题、一定程度上提高模态识别精度, 且更利于自动化。
\end{abstract}

关键词：运行模态分析；传递率测量；伪频响函数矩阵；谐波模态

中图分类号: TB123

\section{Operational Modal Analysis Method Based on the Pseudo Frequency Response Function Matrix}

\section{CHEN Tao ${ }^{1,2}$ CHU Zhigang ${ }^{1}$ LI Peiran ${ }^{2} \quad$ PENG Chuan $^{2} \quad$ YANG Liang $^{1}$}

(1. College of Mechanical and Vehicle Engineering, Chongqing University, Chongqing 400044;

2. China Automotive Engineering Research Institute Corporation Limited, Chongqing 401122)

\begin{abstract}
The operational modal analysis (OMA) technique based on the pseudo frequency response function (PFRF) method by the use of transmissibility measurements does not require the white noise excitation assumption, and has strong ability of harmonic modes rejection. However, its modal identification results depend on the selection of the PFRF to be analyzed, the stability needs to be improved. To solve this issue, an OMA method based on the pseudo frequency response function matrix (PFRFM) method is proposed. Firstly, the PFRFM with multiple reference measurement points and multiple reference loading conditions is constructed. Subsequently, the least squares complex frequency domain method is applied to the PFRFM to extract the modal frequencies and damping ratios, the transmissibility matrices are used to extract the modal shapes. Finally, the number of modes and the final modal results are determined by combing the stabilization diagram with the modal indicator function that is built on the arithmetic mean of the reciprocal of the second singular values about the transmissibility matrices with different references. The results of simulations for a 5 DOF mass-spring-damping system and experiments for a free beam demonstrate that the proposed method not only inherits the strong ability of harmonic modes rejection, but also can effectively solve the problem of insufficient stability of identification results of the PFRF method, obtain higher precision modal results to a certain extent, and is more conducive to automation.
\end{abstract}

Key words: operational modal analysis; transmissibility measurements; pseudo frequency response function matrix; harmonic modes

\section{0 前言}

运行模态分析 (Operational modal analysis,

\footnotetext{
* 国家自然科学基金资助项目(11704040)。20210529 收到初稿, 20210924 收到修改稿
}

OMA)方法由于具有无需停机测量、有助于揭示结 构在真实边界条件下的动力学特性等优点, 近年来 备受关注 ${ }^{[1-3]}$ 。然而当采用基于白噪声激励假设的经 典 OMA 方法时, 实际结构振动信号中存在的谐振 动响应成分可能会被错误地识别成模态(为阐述方 便，后文统一称为 “谐波模态”), 对模态参数的准 确提取造成极大干扰 ${ }^{[4-6]}$ 。针对 “如何识别和剔除谐 
波模态”, 国内外学者进行了大量研究。文献[7]认 为阻尼比为零的模态为谐波模态, 然而该方法对小 阻尼系统并不适用, 且有时谐波模态阻尼比识别结 果并不为零。文献[8]通过对响应信号进行滤波预处 理以剔除混入的谐波信号, 但该方法需提前知晓信 号中的谐波频率, 且当谐波频率与模态频率相近时 容易导致 “过剔除”。文献[9-10]指出可对响应信号 进行带通滤波并计算其概率密度函数(Probability density function, PDF)来区分谐波模态和真实模态, 其中谐波响应的 PDF 曲线为双峰值、共振响应的 PDF 曲线为单峰值, 然而该方法难以实现自动化, 且无法识别阻尼较大的谐波。计算响应信号的峭度 (Kurtosis)亦可区分谐波模态和真实模态, 真实模态 处的峭度约为 3、谐波模态处的峭度为 1.5 , 但该方 法同样无法识别阻尼较大的谐波 ${ }^{[10-11]}$ 。

上述方法依然基于白噪声激励假设, 文 献[12-13]提出一种无需白噪声激励假设的基于传 递率测量的 OMA 方法, 其基于 “传递率函数仅 在系统极点处等于两测点模态振型分量之比、与 激励条件无关” 这一性质, 利用两工况、两测点 的响应数据构建 “在系统极点处将会出现峰值、 在谐波处不会出现峰值” 的伪频响函数并采用频 域参数识别方法对其进行曲线拟合进而获得模态 参数 ${ }^{[14-15]}$ 。该方法后来被称为伪频响函数法, 能 彻底抑制谐波模态, 近年来得到广泛研究与应用。 伪频响函数法的关键在于伪频响函数的构建, 由 于选择不同的工况和测点可构建不同的伪频响函 数, 文献[16]指出基于不同的伪频响函数获得的模 态结果稳定性有待提升, 需谨慎选择才可避免丢 失模态信息。为提升方法识别结果的稳定性, 文 献[16]通过设计更多的工况, 利用所有工况、所有 测点的数据信息重新构建包含了更多信息来源的 改进型伪频响函数, 改善了原始伪频响函数极点 信息凸显程度不足的问题。然而选择不同的参考 测点与参考工况同样可构建得到不同的改进型伪 频响函数, 作者通过大量仿真发现, 基于不同的 改进型伪频响函数获得的模态结果仍面临稳定性 不足的问题。为进一步缓解伪频响函数法的稳定 性问题, 本文建立了伪频响函数矩阵法, 即首先 在改进型伪频响函数基础上构造多参考测点、多 参考工况的伪频响函数矩阵, 然后基于伪频响函 数矩阵采用最小二乘复频域 (Least-squares complex frequency-domain, LSCF) 法求解模态频 率和阻尼比、基于传递率函数矩阵求解模态振型,
最后建立稳态图并结合以多参考测点平均的传递 率函数矩阵第 2 阶奇异值的倒数为基础的模态指 示函数来确定模态阶数及最终的模态结果。该方 法通过进一步充分利用所有工况与测点的信息, 能有效提升识别结果的稳定性, 并能在一定程度 上提高模态识别精度, 且继承了伪频响函数法的 强 “谐波模态抑制” 能力, 无需人为选择伪频响 函数亦有利于自动化，但仍未克服基于传递率测 量的伪频响函数法无法识别重根模态的不足。

\section{1 传递率函数及其矩阵的性质}

假设线性时不变系统受到载荷工况 $A$ 的激励, 则测点 $i$ 与参考测点 $r$ 的传递率函数 $T_{i, r}^{A}(f)$ 定义为 复响应 $X_{i}^{A}(f)$ 与 $X_{r}^{A}(f)$ 的比值, 即 $T_{i, r}^{A}(f)=$ $X_{i}^{A}(f) / X_{r}^{A}(f)$ 。在工程实际中传递率函数通常采用 $H_{1}$ 估 计 ${ }^{[13]}$, 即 $H_{1}^{A}(f)=S_{X_{i} X_{r}}^{A}(f) / S_{X_{r} X_{r}}^{A}(f)$, 其中 $S_{X_{i} X_{r}}^{A}(f)$ 是响应 $X_{i}^{A}(f)$ 和 $X_{r}^{A}(f)$ 的互功率谱、 $S_{X_{r} X_{r}}^{A}(f)$ 是响应 $X_{r}^{A}(f)$ 的自功率谱。可以证明, 在系 统的第 $s$ 阶模态频率 $f_{s}$ 处, 任意载荷工况下的传递 率函数 $T_{i, r}^{A}\left(f_{s}\right)$ 均等于测点 $i$ 和参考测点 $r$ 的模态振 型分量之比、与载荷无关，如式(1)所示

$$
\lim _{f \rightarrow f_{s}} T_{i, r}^{A}(f)=\lim _{f \rightarrow f_{s}} T_{i, r}^{B}(f)=\cdots=\phi_{i}^{(s)} / \phi_{r}^{(s)}
$$

式中, $\phi_{i}^{(s)}$ 和 $\phi_{r}^{(s)}$ 分别是测点 $i$ 和 $r$ 的第 $s$ 阶模态振型 分量。而在非模态频率处式(1)并不成立。

通过改变激励条件设计 $N_{\mathrm{w}}$ 种载荷工况, 则 $N_{\mathrm{w}}$ 种工况、 $N_{\mathrm{o}}$ 个测点可获得 $N_{\mathrm{w}} \times N_{\mathrm{o}}$ 个关于参考测点 $r$ 的传递率函数, 进而可构造出参考测点 $r$ 的传递率 函数矩阵 $\boldsymbol{T}_{r}(f) \in \mathbb{C}^{N_{0} \times N_{w}},\left(r=1,2, \cdots, N_{\mathrm{o}}\right)$

$$
\boldsymbol{T}_{r}(f)=\left(\begin{array}{cccc}
T_{1, r}^{1}(f) & T_{1, r}^{2}(f) & \cdots & T_{1, r}^{N \mathrm{w}}(f) \\
T_{2, r}^{1}(f) & T_{2, r}^{2}(f) & \cdots & T_{2, r}^{N_{\mathrm{w}}}(f) \\
\vdots & \vdots & & \vdots \\
T_{N_{\mathrm{o}}, r}^{1}(f) & T_{N_{\mathrm{o}}, r}^{2}(f) & \cdots & T_{N_{\mathrm{o}}, r}^{N_{\mathrm{w}}}(f)
\end{array}\right)
$$

联立式(1)和式(2)可得

$$
\lim _{f \rightarrow f_{s}} \boldsymbol{T}_{r}(f)=\frac{1}{\phi_{r}^{(s)}}\left(\begin{array}{cccc}
\phi_{1}^{(s)} & \phi_{1}^{(s)} & \cdots & \phi_{1}^{(s)} \\
\phi_{2}^{(s)} & \phi_{2}^{(s)} & \cdots & \phi_{2}^{(s)} \\
\vdots & \vdots & & \vdots \\
\phi_{N_{\mathrm{o}}}^{(s)} & \phi_{N_{\mathrm{o}}}^{(s)} & \cdots & \phi_{N_{\mathrm{o}}}^{(s)}
\end{array}\right)
$$

由式(3)知, 在模态频率处传递率函数矩阵的秩为 1 。 


\section{2 伪频响函数法}

\section{1 模态参数的估计}

\subsection{1 基于伪频响函数的模态频率与阻尼比估计}

基于上述传递率函数及其矩阵的性质，在式(2) 所示传递率函数矩阵中任选两工况下测点 $i$ 与参考 测点 $r$ 的传递率函数 $T_{i, r}^{A}(f)$ 与 $T_{i, r}^{B}(f)$, 并计算这两个 传递率函数之差的倒数 $\Delta^{-1} T_{i, r}^{A, B}(f)$

$$
\Delta^{-1} T_{i, r}^{A, B}(f)=\left(T_{i, r}^{A}(f)-T_{i, r}^{B}(f)\right)^{-1}
$$

由式 (1) 和式 (4) 可知, 当 $f \rightarrow f_{s}$ 时, $\lim _{f \rightarrow f_{s}}\left(\Delta^{-1} T_{i, r}^{A, B}(f)\right) \rightarrow \infty$ 。即 $\Delta^{-1} T_{i, r}^{A, B}(f)$ 在系统固有频 率处会出现很大的峰值, 这与频响函数类似, 因此 $\Delta^{-1} T_{i, r}^{A, B}(f)$ 又被称为伪频响函数 ${ }^{[17]}$, 可用于识别模 态参数; 该函数在谐波频率处不会出现峰值, 因此 能有效抑制谐波模态。

系统极点可通过参数化估计伪频响函数获 得 ${ }^{[12-13]}$, 实质为曲线拟合技术, 通过最小化测量数 据与数学模型间的误差函数来获得系统极点, 进而 获得相应的模态频率与阻尼比。使用伪频响函数的 公分母模型, 则测量数据与数学模型之间的误差函 数 $\varepsilon\left(\omega_{k}\right)$ 定义为

$$
\varepsilon\left(\omega_{k}\right)=\Delta^{-1} T_{i, r}^{A, B}\left(\omega_{k}\right)-\frac{B\left(\omega_{k}, \boldsymbol{\theta}\right)}{A\left(\omega_{k}, \boldsymbol{\theta}\right)}
$$

式中, $\omega_{k}=2 \pi f_{k}$ 为离散圆频率 $\left(k=1,2, \cdots, N_{k}, N_{k}\right.$ 为 频率总数、对应于功率谱的谱线数), $A\left(\omega_{k}, \boldsymbol{\theta}\right)$ 、 $B\left(\omega_{k}, \boldsymbol{\theta}\right)$ 分别为公分母模型的分母、分子多项式, $\boldsymbol{\theta}$ 为待求解的未知参数。由于 LSCF 法相比于其他频 域参数识别方法能有效避免数值求解的病态问 题 ${ }^{[15]}$, 因此本文选择该方法作为提取系统模态频率 与阻尼比的参数识别方法。

\subsection{2 基于传递率函数矩阵的模态振型估计}

根据选定的参考测点 $r$ 构建相应的传递率函数 矩阵 $\boldsymbol{T}_{r}(f)$, 对其进行奇异值分解, 提取与求得的模 态频率 $f_{s}$ 相对应的左奇异矩阵的第 1 列向量 $\boldsymbol{U}_{r}^{(1)}\left(f_{s}\right)$ 即为对应模态阶次的模态振型估计 ${ }^{[18]}$ 。

\section{2 模态阶数与模态参数的确定}

采用 LSCF 法识别模态参数往往需要过估计模 型阶次以确保识别出所有模态, 但过估计模型阶次 不可避免会引入额外的计算极点进而对模态阶数与 模态参数的确定造成干扰。为便于自动化且准确地 定阶并确定最终的模态参数, 稳态图技术 ${ }^{[19]}$ 被引 入。通过改变模型阶次, 根据不同模型阶次下获得 的模态频率、阻尼比及振型建立相应稳态图并提取
最终的模态结果。稳态图标示流程可参考文献[3]。

由于伪频响函数 $\Delta^{-1} T_{i, r}^{A, B}(f)$ 的分母阶次大于系 统频响函数的阶次，当采用 LSCF 法参数识别伪频 响函数得到真实极点的同时亦会产生虚假极点，并 且这些虚假极点可能仍会以稳定极点的形式出现在 稳态图中 ${ }^{[12,20]}$, 影响模态阶数与模态参数的确定。 由传递率函数矩阵的性质可知, 在模态频率 $f_{s}$ 处传 递率函数矩阵 $\boldsymbol{T}_{r}\left(f_{s}\right)$ 的秩为 1 , 此时除了矩阵 $\boldsymbol{T}_{r}\left(f_{s}\right)$ 的第 1 阶奇异值 $\sigma_{r}^{(1)}\left(f_{s}\right)$ 不为 0 以外, 理论上其余奇 异值均应等于 $0($ 实际中接近 0$)$ 。因此, 取其第 2 阶 奇异值 $\sigma_{r}^{(2)}(f)$ 的倒数 $1 / \sigma_{r}^{(2)}(f)$, 则该函数在模态频 率处将会出现峰值、非模态频率处不会出现峰值, 峰值个数即对应模态阶数, 可作为模态指示函数用 以指示模态，将其与稳态图相结合有助于剔除可能 出现的稳定虚假极点并增加识别结果的置信度。

\section{3 识别结果的稳定性分析}

由式(4)可知, 选择不同的工况和测点可获得不 同的伪频响函数 $\Delta^{-1} T_{i, r}^{A, B}(f)$, 文献[16]指出基于不同 的伪频响函数获得的模态结果稳定性有待提升, 需 谨慎选择才可避免丢失真实极点。为此，文献[16] 通过引入全部工况和响应测点的数据信息重新构造 了如式(6)所示关于参考测点 $r$ 、参考工况 $R$ 的改进 型伪频响函数 $\Delta^{-1} T_{\cdot, r}^{*}, R(f)$

$$
\Delta^{-1} T_{\bullet, r}^{\cdot, R}(f)=\left(\sum_{k=1}^{N_{\mathrm{w}}} \sum_{i=1}^{N_{\mathrm{o}}}\left(T_{i, r}^{k}(f)-T_{i, r}^{R}(f)\right)\right)^{-1}
$$

$\Delta^{-1} T_{, r}^{\circ, R}(f)$ 利用了全部载荷工况和响应测点的信息, 能够改善仅利用两种工况、两个测点构造伪频响函 数带来的极点信息凸显程度不足的问题，可获得较 $\Delta^{-1} T_{i, r}^{A, B}(f)$ 更为稳定的模态结果。

下文以图 1 所示 5 自由度质量-弹簧-阻尼系统 为仿真算例进行说明, 其中, 质量 $m_{1}=m_{2}=m_{3}=$ $m_{4}=m_{5}=2 \mathrm{~kg}$ 、弹簧刚度 $k_{1}=k_{2}=k_{3}=k_{4}=k_{5}=$ $1.16 \times 10^{6} \mathrm{~N} / \mathrm{m}$, 阻尼系数分别为 $c_{1}=32 \mathrm{~N} \cdot \mathrm{s} / \mathrm{m}$ 、 $c_{2}=36 \mathrm{~N} \cdot \mathrm{s} / \mathrm{m} 、 c_{3}=40 \mathrm{~N} \cdot \mathrm{s} / \mathrm{m} 、 c_{4}=44 \mathrm{~N} \cdot \mathrm{s} / \mathrm{m}$ 、 $c_{5}=48 \mathrm{~N} \cdot \mathrm{s} / \mathrm{m}, x_{n}$ 为各自由度位移 $(n=1,2, \cdots, 5)$ 。 特征值法提取该系统的模态参数, 其模态频率、阻 尼比的理论值如表 1 所示, 以质量单元 1 归一化的 复模态振型的理论值如表 2 所示。

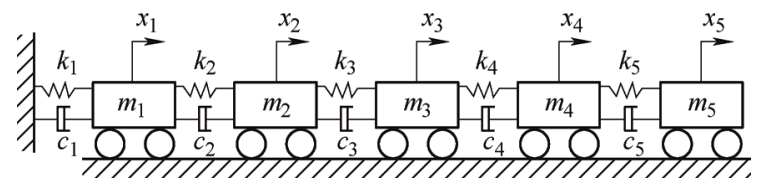

图 15 自由度质量-弹簧-阻尼系统 
表 15 自由度质量-弹簧-阻尼系统的理论模态参数

\begin{tabular}{ccc}
\hline 阶次 & 模态频率 $/ \mathrm{Hz}$ & 阻尼比 $(\%)$ \\
\hline 1 & 34.5 & 0.34 \\
2 & 100.7 & 1.11 \\
3 & 158.7 & 1.76 \\
4 & 203.9 & 2.26 \\
5 & 232.5 & 2.58 \\
\hline
\end{tabular}

表 25 自由度质量-弹簧-阻尼系统的理论振型

\begin{tabular}{cccccc}
\hline & \multicolumn{5}{c}{ 质量单元 } \\
\cline { 2 - 6 } & $m_{1}$ & $m_{2}$ & $m_{3}$ & $m_{4}$ & $m_{5}$ \\
\hline 1 & 1.00 & $1.92-0.00 \mathrm{i}$ & $2.68-0.00 \mathrm{i}$ & $3.23-0.00 \mathrm{i}$ & $3.51-0.00 \mathrm{i}$ \\
2 & 1.00 & $1.31-0.00 \mathrm{i}$ & $0.72-0.01 \mathrm{i}$ & $-0.37-0.00 \mathrm{i}$ & $-1.20+0.01 \mathrm{i}$ \\
3 & 1.00 & $0.28-0.01 \mathrm{i}$ & $-0.92-0.00 \mathrm{i}$ & $-0.55+0.01 \mathrm{i}$ & $0.76-0.00 \mathrm{i}$ \\
4 & 1.00 & $-0.83-0.02 \mathrm{i}$ & $-0.31+0.03 \mathrm{i}$ & $1.09-0.08 \mathrm{i}$ & $-0.59-0.00 \mathrm{i}$ \\
5 & 1.00 & $-1.68-0.03 \mathrm{i}$ & $1.83+0.07 \mathrm{i}$ & $-1.40-0.08 \mathrm{i}$ & $0.52+0.04 \mathrm{i}$ \\
\hline
\end{tabular}

通过改变激励性质设计了两种仿真案例, 其中 案例 1:5 个不相干零均值高斯白噪声分别同步施加 于 5 个质量单元上, 通过改变高斯白噪声均方值设 计了 5 种载荷工况, 各工况白噪声激励的均方值分 布如图 2a 所示, 图 $2 b$ 为施加于质量单元 1 上的各 白噪声激励的自谱(以单位值 $1 \mathrm{~N}^{2} / \mathrm{Hz}$ 为参考进行 $\mathrm{dB}$ 缩放); 案例 2 在案例 1 的 5 种工况基础上添加 了图 2c 所示的简谐激励信号 (简谐激励频率均为 $50 \mathrm{~Hz}$ ), 以验证伪频响函数法抑制谐波模态的能力。

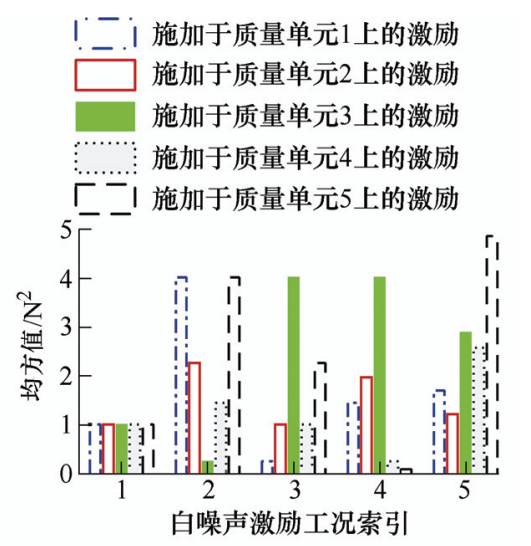

(a) 白噪声激励的均方值分布

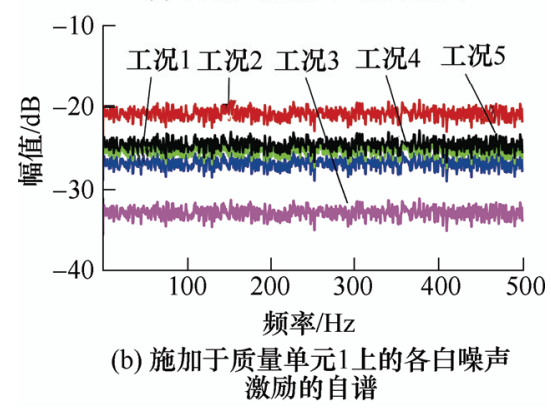

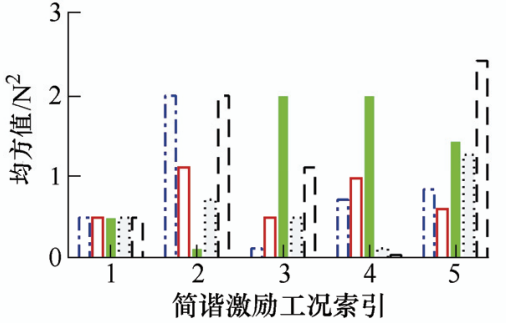

(c) 简谐激励的均方值分布

图 2 两种仿真案例各载荷工况激励

采样频率设为 $1024 \mathrm{~Hz}$, 测量时长 $50 \mathrm{~s}$, 傅里 叶变换时的参数设置为: 频率分辨率 $0.5 \mathrm{~Hz}$ 、汉宁 窗、重叠率 $50 \%$, 数据块总数为 49 且采用线性平均 方式进行平均, 响应信号中未添加测量噪声。分别 提取两种案例下各工况所有测点的位移响应, 以每 个测点分别作为参考构建传递率函数矩阵 $\boldsymbol{T}_{r}(f) \in \mathbb{C}^{5 \times 5}, \quad(r=1,2, \cdots, 5)$, 下文选择以测点 2 和 测点 4 为参考为例进行分析对比。图 3 为两种案例 下伪频响函数法以不同的伪频响函数为数据输入获 得的稳态图, 其中图 3a 3d 以原始伪频响函数 $\Delta^{-1} T_{i, r}^{A, B}(f)$ 为数据输入, 图 $3 \mathrm{e} \sim 3 \mathrm{~h}$ 以重新构造的伪 频响函数 $\Delta^{-1} T_{., r}^{0, R}(f)$ 为数据输入。建立稳态图时模 型阶次区间设为 [20,70]、连续两阶模型识别出的模 态参数稳定性判据分别为: 模态频率变化率小于 $1 \%$ 、阻尼比的变化率小于 $10 \%$ 、振型之间的互模态 置信准则(Cross modal assurance criterion, Cross $\mathrm{MAC})^{[21]}$ 大于 0.98 。本文后续仿真与试验的稳态图 均采用相同的稳定性判据，不再赘述。

由图 3a 3d 可见, 两种案例各自对应的模态 指示函数(第 2 阶奇异值的倒数 $1 / \sigma_{2}^{(2)}(f)$ 与 $1 / \sigma_{4}^{(2)}(f)$ ) 的峰值均与系统固有频率的理论值相对应, 准确 指示出全部 5 阶模态, 且未反映出谐波模态信息。 结合模态指示函数可知, 以 $\Delta^{-1} T_{1,2}^{2,1}(f)$ 为数据输入 获得的稳态图(图 3a 和图 3b)均仅识别出系统的 3 阶模态, 第 1 阶与第 3 阶模态未被识别; 而以 $\Delta^{-1} T_{1,4}^{1,5}(f)$ 为数据输入获得的稳态图(图 $3 \mathrm{c}$ 和图 $3 \mathrm{~d}$ ) 则准确识别出 5 阶模态, 且抑制了谐波模态, 但 案例 2 中第 1 阶稳定极点识别结果不理想(图 3d)。 以 $\Delta^{-1} T_{0,2}^{*, 1}(f)$ 为数据输入获得的稳态图 (图 $3 \mathrm{e}$ 和 图 3f) 相较于以 $\Delta^{-1} T_{1,2}^{2,1}(f)$ 为数据输入获得的稳态 图(图 3a 和图 3b)有所改善, 但仍无法识别出全部 5 阶模态, 第 2 阶模态未被识别, 案例 1 中还识别 出了 1 阶稳定虚假极点(图 $3 \mathrm{e}$ )。以 $\Delta^{-1} T_{, 4}^{, 5}(f)$ 为数 据输入获得的稳态图(图 $3 \mathrm{~g}$ 和图 3h) 结果最为理 想, 既准确识别出了系统的 5 阶模态, 亦有效抑 
制了谐波模态。

上述结果表明: (1) 伪频响函数法能有效识别结 构模态、抑制谐波模态，但基于不同的伪频响函数 $\Delta^{-1} T_{i, r}^{A, B}(f)$ 获得的模态结果稳定性欠佳, 工况和测 点的选择对模态结果的完整获取有显著影响; (2) 通 过引入全部工况和响应测点的数据信息重新构造改 进型伪频响函数 $\Delta^{-1} T_{\bullet, r}^{*, R}(f)$ 能一定程度上缓解原始
伪频响函数极点信息凸显程度不足的问题，但其实 质仍然是对单参考工况 $R$ 单参考测点 $r$ 的标量函数 进行曲线拟合来获得模态结果, 选择的 $\Delta^{-1} T_{., r}^{\cdot, R}(f)$ 函数仍可能无法反映系统所有模态。综上所述, 无 论采用原始伪频响函数还是改进型伪频响函数, 均 需要人为选择参考测点与参考工况、均面临识别结 果稳定性欠佳问题。

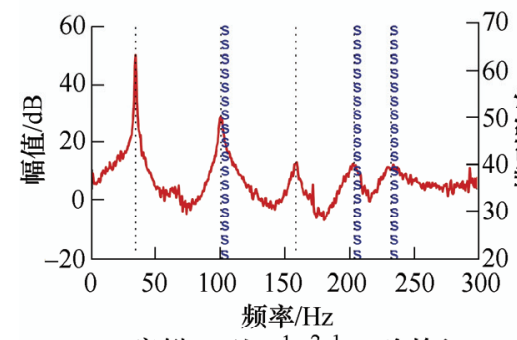

(a) 案例 $1:$ 以 $\Delta^{-1} T_{1,2}^{2,1}(f)$ 为输入

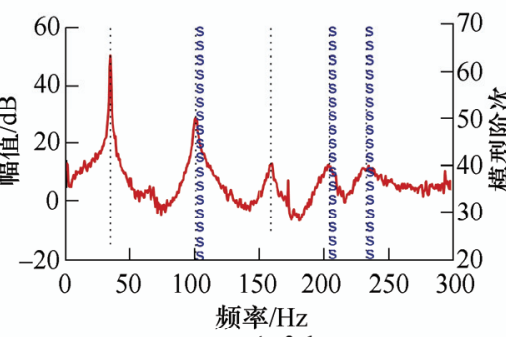

(b) 案例 2: 以 $\Delta^{-1} T_{1,2}^{2,1}(f)$ 为输入

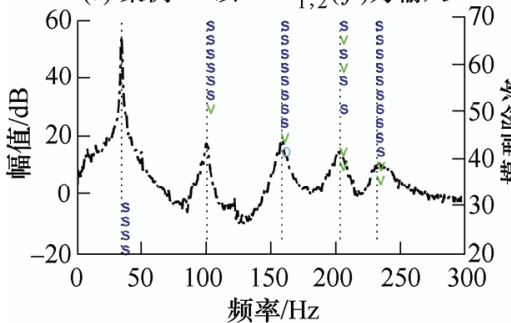

(d) 案例 $2:$ 以 $\Delta^{-1} T_{1,4}^{1,5}(f)$ 为输入

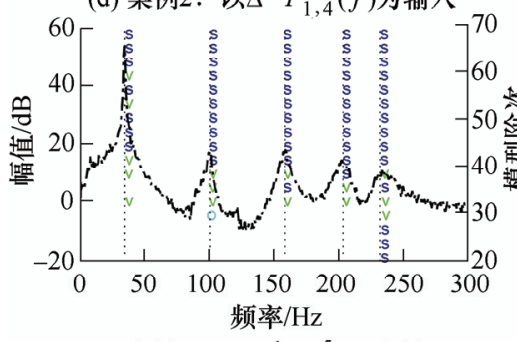

(g) 案例 $1:$ 以 $\Delta^{-1} T \cdot,{ }_{4}^{5}(f)$ 为输入

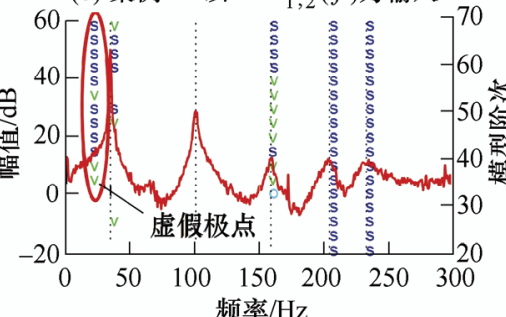

(e) 案例 $1:$ 以 $\Delta^{-1} T:{ }_{2}^{1}(f)$ 为输入

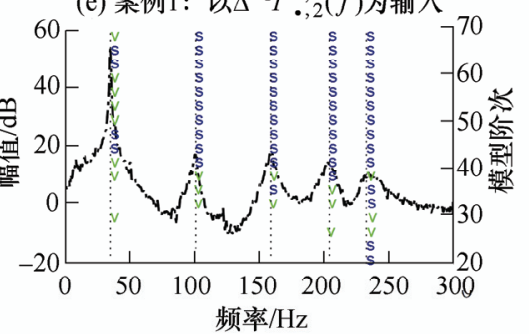

(h) 案例 $2:$ 以 $\Delta^{-1} T: 54$, $(f)$ 为输入

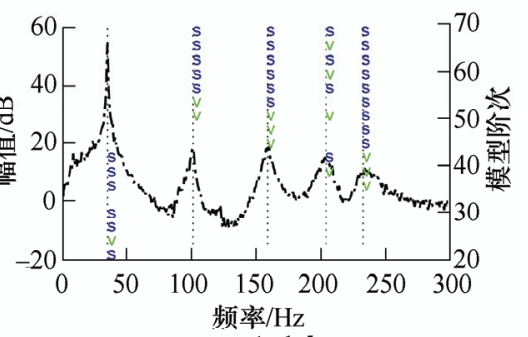

(c) 案例 1 : 以 $\Delta^{-1} T_{1,4}^{1,5}(f)$ 为输入



(f) 案例 $2:$ 以 $\Delta^{-1} T:, 2(f)$ 为输入

$1 / \sigma_{4}^{(2)}(f) \quad-\cdot-1 / \sigma_{4}^{(2)}(f)$ 固有频率的理论值

频率、阻尼和振型均稳定

频率与振型稳定

频率、阻尼和振型均不稳定

图 3 两种仿真案例伪频响函数法以不同伪频响函数为输入获得的稳态图

\section{3 伪频响函数矩阵法}

为进一步缓解伪频响函数法结果稳定性欠佳这 一问题，本文在式(6)基础上首先构造多参考工况、 多参考测点的伪频响函数矩阵, 然后采用 LSCF 法 以该矩阵为基础进行模态参数识别, 建立了伪频响 函数矩阵法。

\section{1 模态参数的估计}

3.1.1 基于伪频响函数矩阵的模态频率与阻尼比估计 选择所有测点和工况分别作为参考, 则可获得 $N_{\mathrm{w}} \times N_{\mathrm{o}}$ 个如式(6)所示的伪频响函数 $\Delta^{-1} T_{0, r}^{*, R}(f)$, $\left(R=1,2, \cdots, N_{\mathrm{w}} 、 r=1,2, \cdots, N_{\mathrm{o}}\right)$, 进一步可构造式(7) 所示多参考测点、多参考工况的伪频响函数矩阵 $\Delta^{-1} \boldsymbol{T}(f) \in \mathbb{C}^{N_{\mathrm{o}} \times N_{\mathrm{w}}}$, 且

\section{$\Delta^{-1} \boldsymbol{T}(f)=$}

$$
\left(\begin{array}{cccc}
\Delta^{-1} T_{,, 1}^{\cdot, 1}(f) & \Delta^{-1} T_{,, 1}^{\cdot, 2}(f) & \cdots & \Delta^{-1} T_{, 1}^{, N_{\mathrm{w}}}(f) \\
\Delta^{-1} T_{, 2}^{\cdot, 1}(f) & \Delta^{-1} T_{, \cdot 2}^{\cdot, 2}(f) & \cdots & \Delta^{-1} T_{,, 2}^{\cdot, N_{\mathrm{w}}}(f) \\
\vdots & \vdots & & \\
\Delta^{-1} T_{\cdot, N_{\mathrm{o}}}^{\cdot, 1}(f) & \Delta^{-1} T_{\cdot, N_{\mathrm{o}}}^{\cdot, 2}(f) & \cdots & \Delta^{-1} T_{\cdot, N_{\mathrm{o}}}^{\cdot, N_{\mathrm{w}}}(f)
\end{array}\right)
$$

与频响函数矩阵类似, 该矩阵中所有元素在系 统固有频率处均会出现很大的峰值, 采用 LSCF 法 对该矩阵整体进行模态参数识别即可获得模态频率 与阻尼比。其充分利用了所有 $\Delta^{-1} T_{, r}, r(f)$ 函数的数 据, 能进一步避免以单一 $\Delta^{-1} T_{0, r}^{*, R}(f)$ 函数为数据输 入可能导致模态丢失的情况出现, 无需人为选择伪 频响函数亦有利于自动化。同时, 该矩阵亦继承了 强 “谐波模态抑制” 能力。采用伪频响函数矩阵的 右矩阵分式模型, 测量数据与数学模型间的误差函 
数 $\boldsymbol{\varepsilon}\left(\omega_{k}, \boldsymbol{\theta}\right)$ 为

$$
\boldsymbol{\varepsilon}\left(\omega_{k}, \boldsymbol{\theta}\right)=\Delta^{-1} \boldsymbol{T}\left(\omega_{k}\right)-\boldsymbol{B}\left(\omega_{k}, \boldsymbol{\theta}\right) \boldsymbol{A}^{-1}\left(\omega_{k}, \boldsymbol{\theta}\right)
$$

式中, $\boldsymbol{B}\left(\omega_{k}, \boldsymbol{\theta}\right) \in \mathbb{C}^{N_{\mathrm{o}} \times N_{\mathrm{w}}} 、 \boldsymbol{A}\left(\omega_{k}, \boldsymbol{\theta}\right) \in \mathbb{C}^{N_{\mathrm{w}} \times N_{\mathrm{w}}}$ 分别为右 矩阵分式模型的分子、分母多项式矩阵。

\subsection{2 基于传递率函数矩阵的模态振型估计}

为提升振型识别结果的精度, 本文将所有测点 分别作为参考构造的传递率函数矩阵 $\boldsymbol{T}_{r}(f)$ 均进行 奇异值分解, 提取所有左奇异矩阵的第 1 列向量 $\boldsymbol{U}_{r}^{(1)}(f)$ 并进行归一化处理得到 $\overline{\boldsymbol{U}}_{r}^{(1)}(f)$, 然后求其均 值, 如式(9)所示

$$
\phi(f)=\frac{1}{N_{\mathrm{o}}} \sum_{r=1}^{N_{\mathrm{o}}} \overline{\boldsymbol{U}}_{r}^{(1)}(f)
$$

根据 LSCF 法求出的模态频率 $f_{s}$ 提取对应的 $\phi\left(f_{s}\right)$ 作为模态振型的估计, 其利用了多参考测点平 均, 进而获得的结果精度更高、稳定性更好。

\subsection{3 模态阶数与模态参数的确定}

由图 3 的仿真结果可知, 不同参考测点的模 态指示函数 $1 / \sigma_{r}^{(2)}(f)$ 虽均能准确指示出系统全部 模态频率所在位置, 但亦有所差异, 相对而言, 图 3c、3d、3g、3h 中采用的 $1 / \sigma_{4}^{(2)}(f)$ 函数曲线更 加光滑, 模态指示效果更好。为消除不同参考测
点带来的模态指示效果的差异、避免不恰当的参 考测点选择, 本文重新构造了如式(10)的模态指示 函数, 其通过将所有测点均作为参考测点并求取 相应传递率函数矩阵的第 2 阶奇异值的倒数后加 以平均获得。

$$
\mu(f)=\frac{1}{N_{\mathrm{o}}} \sum_{r=1}^{N_{\mathrm{o}}} \frac{1}{\sigma_{r}^{(2)}(f)}
$$

\section{2 仿真分析}

综上, 所提伪频响函数矩阵法的仿真分析流程 如图 4 所示, 具体实现步骤如下。首先设计 $N_{\mathrm{w}}$ 种载 荷工况, 分别以每个测点作为参考构建相应的传递 率函数矩阵; 然后基于式(6)计算所有伪频响函数, 构建式(7)所示伪频响函数矩阵, 同时对所有传递率 函数矩阵分别进行奇异值分解, 计算式(10)所示模 态指示函数 $\mu(f)$ 以及提取归一化之后的左奇异矩 阵的第 1 列向量并求其均值 $\phi(f)$; 进一步采用 LSCF 法对伪频响函数矩阵整体进行模态参数识别求取系 统极点, 并提取极点对应模态频率的 $\phi\left(f_{s}\right)$ 作为振 型估计，进而建立稳态图并同时绘制模态指示函数 $\mu(f)$ 来指示模态; 最后结合稳态图和模态指示函数 来提取最终的模态参数。

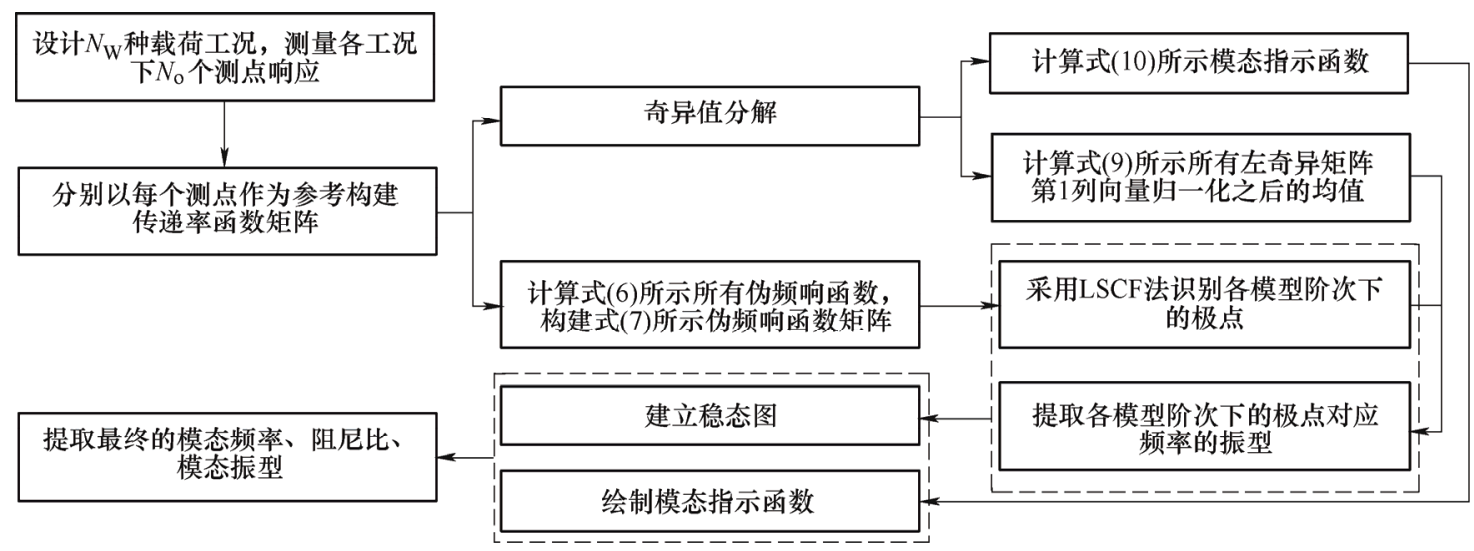

图 4 伪频响函数矩阵法的仿真分析流程

采用伪频响函数矩阵法对第 3 节中的 5 自由度 系统进行模态参数识别, 两种仿真案例下获得的稳 态图如图 5 所示。与图 3 对比可见, 基于多参考测 点平均的模态指示函数(图 5 中曲线) 较单参考测点 的模态指示函数(图 3 中曲线)更加清晰地指示出系 统的全部 5 阶模态。两种案例下基于伪频响函数矩 阵法获得的稳态图(图 5)均准确识别出了所有模态, 亦抑制了谐波模态, 且稳态图较基于伪频响函数法 获得的稳态图(图 3)更优。最后采用参数均值法 ${ }^{[3]}$ 分 别提取基于伪频响函数法与伪频响函数矩阵法获得 的稳态图中的模态结果(伪频响函数法仅展示稳态
图结果最为理想的结果, 即以 $\Delta^{-1} T_{: 4}^{* 5}(f)$ 为输入), 基于两种方法最终获得的模态频率和阻尼比分别如 表 3、4 所示。两种案例下两种方法的识别结果与理 论值间的误差如图 6 所示。图 6 中频率误差定义为 $\Delta f=\left|f_{\text {Estimated }}-f_{\text {Theory }}\right| / f_{\text {Theory }} \times 100 \%$ 、阻尼比误差定 义为 $\Delta \xi=\left|\xi_{\text {Estimated }}-\xi_{\text {Theory }}\right|$ 、振型误差定义为 $\Delta \phi=$ $(1-\operatorname{Cross} M A C) \times 100 \%$, 其中 $f_{\text {Estimated }}$ 和 $\xi_{\text {Estimated }}$ 分别 为模态频率与阻尼比识别结果, $f_{\text {Theory }}$ 和 $\xi_{\text {Theory }}$ 分别 为模态频率与阻尼比理论值, Cross MAC 为同一模 态阶次下识别振型与理论振型的 Cross MAC 值。 


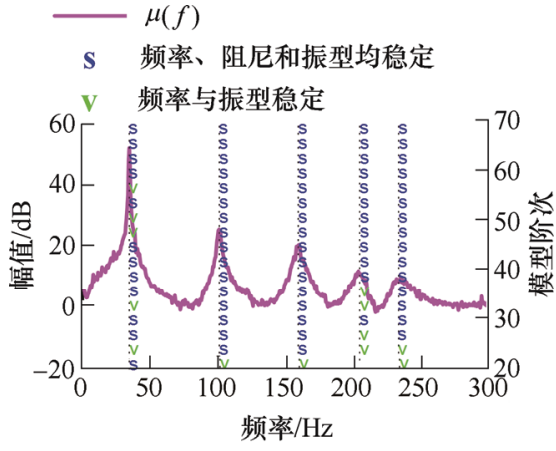

(a) 案例 1

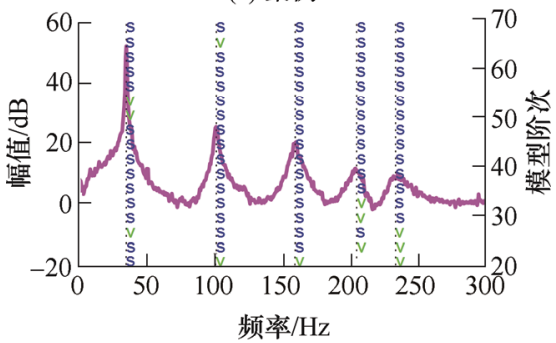

(b) 案例2

图 5 两种仿真案例基于伪频响函数矩阵法获得的稳态图

表 3 基于伪频响函数法的模态频率与阻尼比识别结果

\begin{tabular}{ccccccc}
\hline \multirow{2}{*}{ 阶次 } & \multicolumn{2}{c}{ 案例 1} & & \multicolumn{2}{c}{ 案例 2} \\
\cline { 2 - 3 } \cline { 5 - 6 } \cline { 5 - 6 } & 频率/Hz & 阻尼比 $(\%)$ & & 频率/Hz & 阻尼比 $(\%)$ \\
\hline 1 & 34.7 & 0.34 & & 34.7 & 0.28 \\
2 & 100.2 & 1.47 & & 100.3 & 1.48 \\
3 & 157.6 & 1.16 & & 157.6 & 1.22 \\
4 & 203.4 & 0.84 & & 203.3 & 0.89 \\
5 & 232.8 & 0.67 & & 232.7 & 0.83 \\
\hline
\end{tabular}

表 4 基于伪频响函数矩阵法的模态频率与阻尼比识别结果

\begin{tabular}{cccccc}
\hline \multirow{2}{*}{ 阶次 } & \multicolumn{2}{c}{ 案例 1} & & \multicolumn{2}{c}{ 案例 2} \\
\cline { 2 - 3 } \cline { 5 - 6 } \cline { 5 - 6 } & 频率/Hz & 阻尼比 $(\%)$ & & 频率/Hz & 阻尼比 $(\%)$ \\
\hline 1 & 34.3 & 0.26 & & 34.4 & 0.27 \\
3 & 100.7 & 1.17 & & 100.7 & 1.15 \\
3 & 158.7 & 1.32 & & 158.7 & 1.35 \\
4 & 204.0 & 1.67 & & 204.0 & 1.70 \\
5 & 232.3 & 1.73 & & 232.5 & 1.76 \\
\hline
\end{tabular}

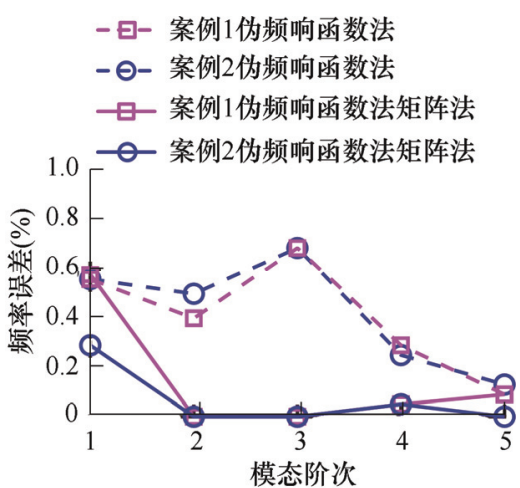

(a) 频率误差

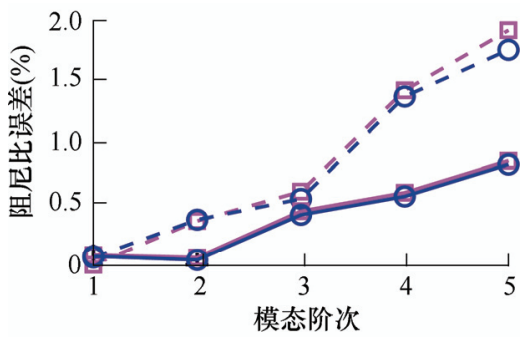

(b) 阻尼比误差

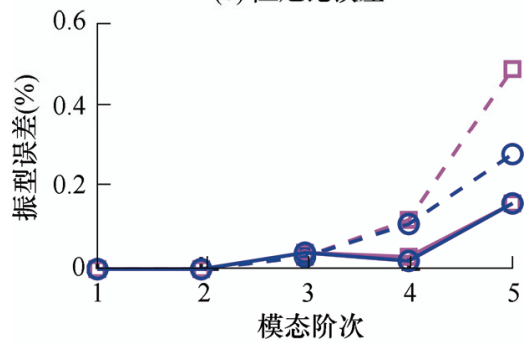

(c) 振型误差

图 6 识别结果与理论值之间的误差

从图 6 可见, 两种案例下两种方法均准确识别 出了系统的 5 阶模态，总体上讲基于伪频响函数矩 阵法获得的模态结果精度更高, 且该方法无需人为 选择伪频响函数，具有明显的优越性。

进一步考虑测量噪声干扰的影响, 以案例 1 为 例, 在其时域响应信号中加入信噪比(Signal to noise ratio, SNR)为 $30 \mathrm{~dB}$ 的随机白噪声来对比两种方法 抗测量噪声干扰的能力。采用两种方法分别获得的 稳态图如图 7 所示, 可以看到伪频响函数法(图 7a) 仅识别出了系统的前 3 阶模态, 而伪频响函数矩阵 法(图 7b)仍然准确识别出了全部 5 阶模态, 可见伪 频响函数法受测量噪声影响较大, 伪频响函数矩阵 法的抗测量噪声干扰能力优于伪频响函数法。

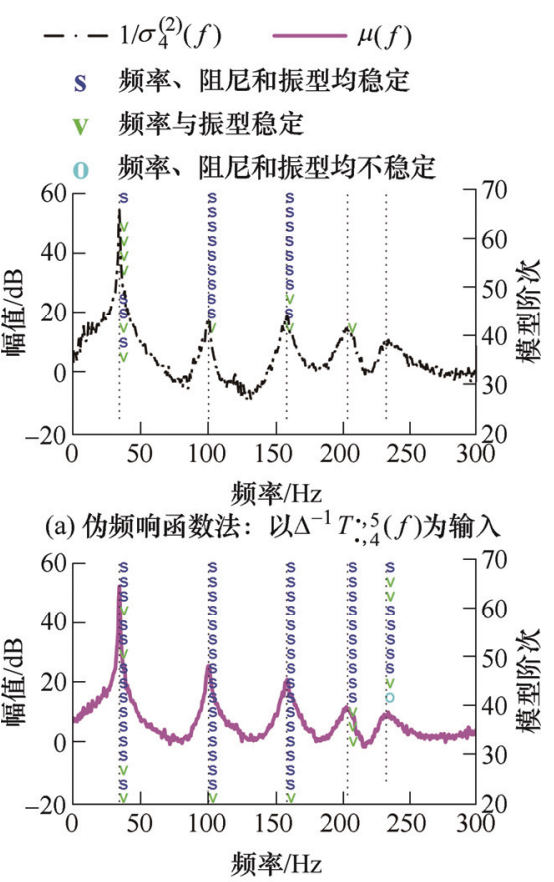

(b) 伪频响函数矩阵法

图 $7 \mathrm{SNR}$ 为 $30 \mathrm{~dB}$ 时的稳态图 


\section{4 试验分析}

为验证本文提出方法的有效性和优越性, 对一 铁质自由梁进行运行模态分析。测量现场及测点布 置如图 8 所示, 自由梁上等间距布置 5 只加速度传 感器, 在 $1 、 2$ 号传感器之间固定一只微电机, 利用 两只力锤连续随机敲击产生随机脉冲激励和利用微 电机运转产生简谐激励, 微电机的工作频率范围为 $300 \sim 333 \mathrm{~Hz}$, 箭头处为力锤敲击点。

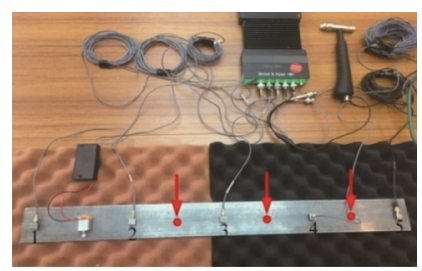

图 8 运行模态试验现场布置图
利用 B\&K 3050 型数据采集系统同时采集所 有加速度传感器的信号, 采样频率为 $8192 \mathrm{~Hz}$, 采样时长 $30 \mathrm{~s}$, 快速傅里叶变换时参数设置为: 频率分辨率 $0.5 \mathrm{~Hz}$ 、汉宁窗、重叠率 $50 \%$, 数据 块总数为 29 且采用线性平均方式进行平均。通过 改变微电机工作状态设计了两种试验案例, 其中 案例 1 为: 微电机未运转, 激励仅由两只力锤连 续随机敲击产生，通过改变力锤敲击位置和力度 设计了 4 种载荷工况; 案例 2 为: 使微电机保持 运转, 改变力锤敲击位置和力度亦设计了 4 种载 荷工况。图 9 为两种案例下伪频响函数法分别以 不同的伪频响函数为数据输入获得的稳态图, 其 中图 $9 \mathrm{a} \sim 9 \mathrm{~d}$ 以原始伪频响函数 $\Delta^{-1} T_{i, r}^{A, B}(f)$ 为数据 输入，图 9e $9 \mathrm{~h}$ 以文献[16]构造的改进型伪频响 函数 $\Delta^{-1} T_{0, r}^{, R}(f)$ 为数据输入。

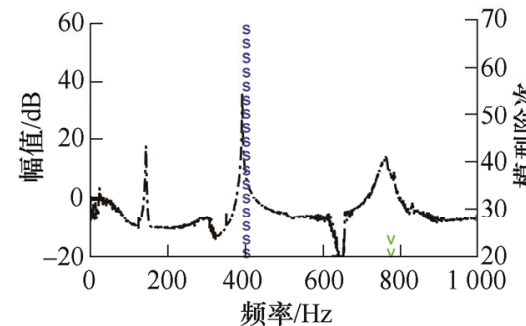

(a) 案例 $1:$ 以 $\Delta^{-1} T_{1,4}^{1,2}(f)$ 为输入

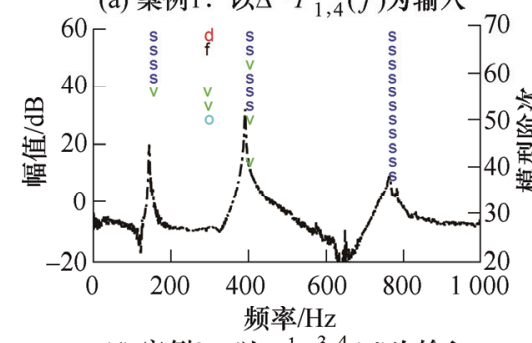

(d) 案例 $2:$ 以 $\Delta^{-1} T_{1,4}^{3,4}(f)$ 为输入

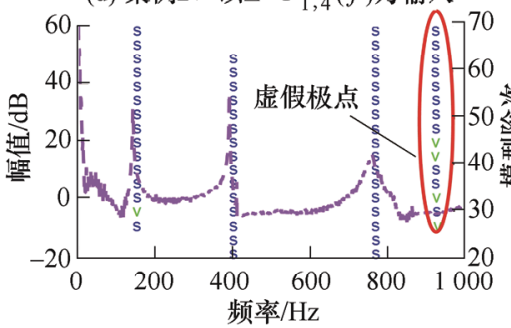

(g) 案例 $1:$ 以 $\Delta^{-1} T::_{5}^{4}(f)$ 为输入

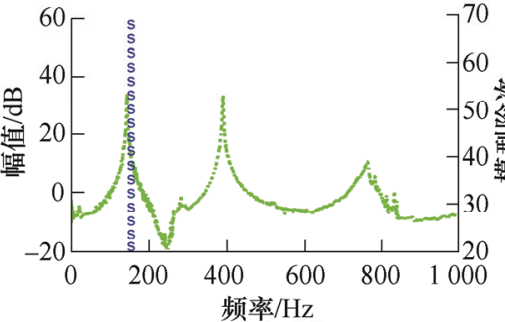

(b) 案例 2 : 以 $\Delta^{-1} T_{4,1}^{3,2}(f)$ 为输入

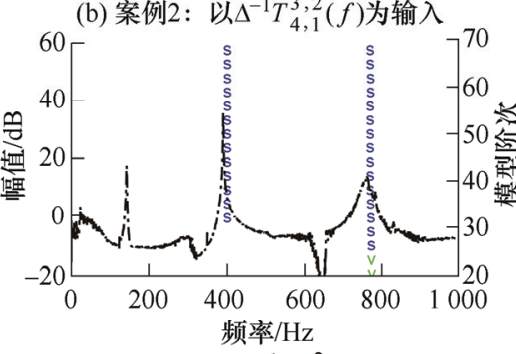

(e) 案例 $1:$ 以 $\Delta^{-1} T^{*}:{ }_{4}^{2}(f)$ 为输入

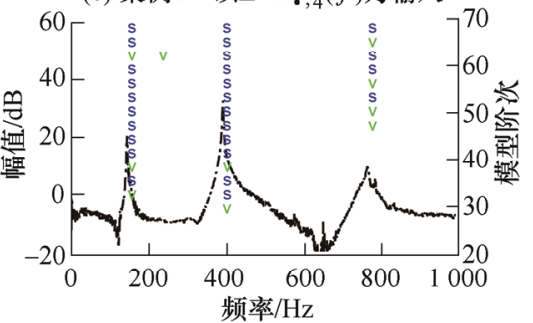

(h) 案例 $2:$ 以 $\Delta^{-1} T \cdot{ }_{5}(f)$ 为输入

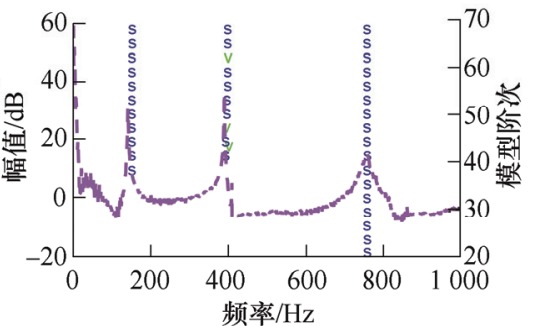

(c) 案例 1: 以 $\Delta^{-1} T_{3,5}^{3,4}(f)$ 为输入

图 9 两种试验案例伪频响函数法以不同伪频响函数为数据输入获得的稳态图

由图 9a 9c 可见, 分别以第 2 阶奇异值的倒数 $1 / \sigma_{1}^{(2)}(f) 、 1 / \sigma_{4}^{(2)}(f)$ 与 $1 / \sigma_{5}^{(2)}(f)$ 为模态指示函数的曲 线均在 $0 \sim 1000 \mathrm{~Hz}$ 频率范围内清晰可见 3 个峰值, 且未在微电机的工作频率处出现峰值, 即模态指示 函数 $1 / \sigma_{r}^{(2)}(f)$ 既有效抑制了简谐激励信息, 亦指示 出该自由梁在 $0 \sim 1000 \mathrm{~Hz}$ 频率范围内存在 3 阶模
态。从图 $9 \mathrm{a} \sim 9 \mathrm{~d}$ 还可知, 两种案例分别以 $\Delta^{-1} T_{1,4}^{1,2}(f) 、 \Delta^{-1} T_{4,1}^{3,2}(f)$ 为输入的稳态图(图 $9 \mathrm{a} 、 9 \mathrm{~b}$ ) 仅识别出自由梁的 1 阶模态, 而分别以 $\Delta^{-1} T_{3,5}^{3,4}(f)$ 、 $\Delta^{-1} T_{1,4}^{3,4}(f)$ 为输入的稳态图(图 9c、9d) 则识别出了自 由梁的前 3 阶模态。由图 9e $\sim 9 \mathrm{~h}$ 可见，相同参考测 点下以重新构造的伪频响函数 $\Delta^{-1} T_{0, r}^{*}, R(f)$ 为输入获 
得的稳态图相较于以原始伪频响函数 $\Delta^{-1} T_{i, r}^{A, B}(f)$ 为 输入获得的稳态图有所改善, 但其仍受参考测点与 参考工况选择的影响: 案例 1 以 $\Delta^{-1} T_{., 4}^{*, 2}(f)$ 为输入获 得的稳态图 (图 9e) 仅识别出两阶模态, 而以 $\Delta^{-1} T_{\cdot, 5}^{*}(f)$ 为输入获得的稳态图(图 $9 \mathrm{~g}$ ) 识别出 4 阶模 态, 其中与模态指示函数的 3 个峰值相对应的为自 由梁的真实模态, 另一为虚假模态; 案例 2 以 $\Delta^{-1} T_{\cdot, 1}^{\cdot, 2}(f)$ 为输入获得的稳态图(图 9f)仅识别出两 阶模态, 而以 $\Delta^{-1} T_{\cdot, 4}^{\cdot 4}(f)$ 为输入获得的稳态图(图 9h) 则识别出了自由梁的前 3 阶模态。以上结果表 明, 伪频响函数法能识别系统模态参数、抑制谐 波模态, 然而无论是以原始伪频响函数还是以文 献[16]构造的改进型伪频响函数为数据输入, 其 模态识别结果稳定性均受人为选择的测点与工 况影响较大。

图 10 为两种试验案例下分别基于所提伪频响 函数矩阵法获得的稳态图, 可见基于多参考测点平 均的模态指示函数 $\mu(f)$ 清晰指示出自由梁的前 3 阶模态, 模态指示效果较单参考测点的模态指示函 数 $1 / \sigma_{r}^{(2)}(f)$ (图 9 中曲线)更优。从模态稳定性判断 结果还可知, 两种案例下所提出方法均准确识别出 了自由梁的所有模态, 亦抑制了微电机运转所带来 的谐波模态。

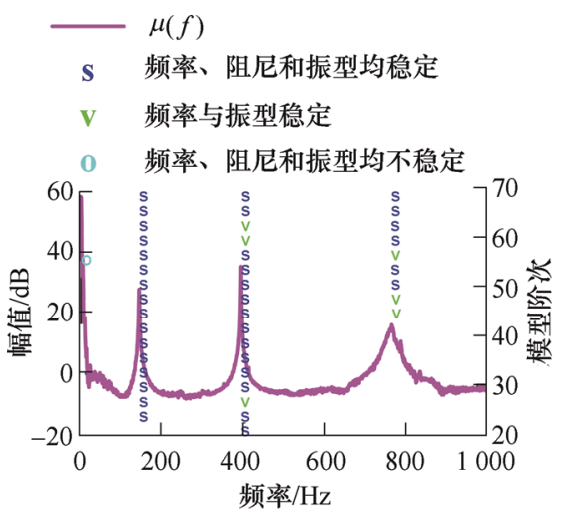

(a) 案例1

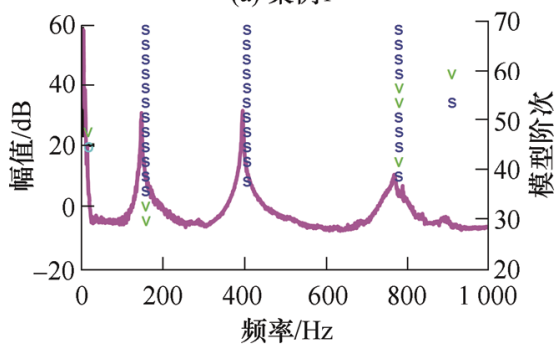

(b) 案例2

图 10 两种试验案例基于伪频响函数矩阵法获得的稳态图

两种案例下分别基于伪频响函数法与伪频响函 数矩阵法获得的模态结果如表 5、6 所示(伪频响函
数法仅展示稳态图最为理想的结果, 即图 $9 \mathrm{~g}$ 与 图 9h)。为验证这些识别结果的准确性, 作者又基于 锤击法对自由梁进行了试验模态分析, 并以锤击法 试验模态的识别结果(表 7)作为参考进行精度度量。 两种方法的识别结果与锤击法试验模态分析结果的 误差如图 11 所示, 由图 11 可知, 两种方法均具有 较高的识别精度, 表明本文提出方法能够获得与文 献[16]构造的伪频响函数法在最优情况下相当的 性能, 且无需选择待分析的伪频响函数进而易于 自动化, 具有明显优势。所得结论与前述仿真结 论一致。

表 5 基于伪频响函数法的模态频率与阻尼比识别结果

\begin{tabular}{cccccc}
\hline \multirow{2}{*}{ 阶次 } & \multicolumn{2}{c}{ 案例 1} & & \multicolumn{2}{c}{ 案例 2} \\
\cline { 2 - 3 } \cline { 5 - 6 } \cline { 5 - 6 } & 频率/Hz & 阻尼比 $(\%)$ & & 频率/Hz & 阻尼比 $(\%)$ \\
\hline 1 & 144.3 & 0.33 & & 144.6 & 0.35 \\
2 & 393.1 & 0.11 & & 392.5 & 0.17 \\
3 & 762.0 & 0.91 & & 765.2 & 0.47 \\
\hline
\end{tabular}

表 6 基于伪频响函数矩阵法的模态频率与阻尼比识别结果

\begin{tabular}{cccccc}
\hline \multirow{2}{*}{ 阶次 } & \multicolumn{2}{c}{ 案例 1} & & \multicolumn{2}{c}{ 案例 2} \\
\cline { 2 - 3 } \cline { 5 - 6 } \cline { 5 - 6 } & 频率/Hz & 阻尼比 $(\%)$ & & 频率/Hz & 阻尼比 $(\%)$ \\
\hline 1 & 144.3 & 0.32 & & 144.6 & 0.31 \\
2 & 393.4 & 0.36 & & 392.3 & 0.17 \\
3 & 763.4 & 0.29 & & 764.9 & 0.24 \\
\hline
\end{tabular}

表 7 锤击法试验模态的模态频率与阻尼比识别结果

\begin{tabular}{ccc}
\hline 阶次 & 频率 $/ \mathrm{Hz}$ & 阻尼比(\%) \\
\hline 1 & 144.0 & 1.31 \\
2 & 393.1 & 0.48 \\
3 & 767.9 & 0.27 \\
\hline
\end{tabular}

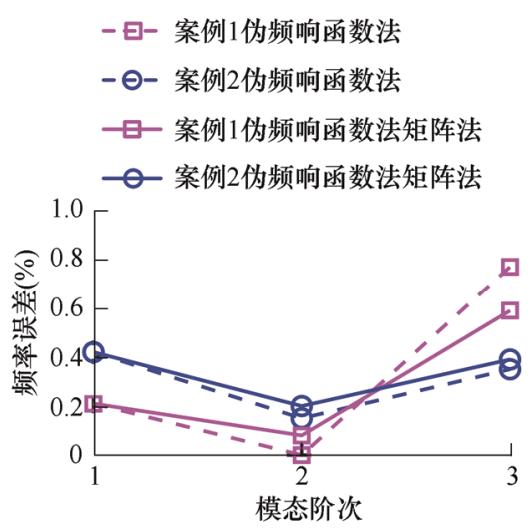

(a) 频率误差 


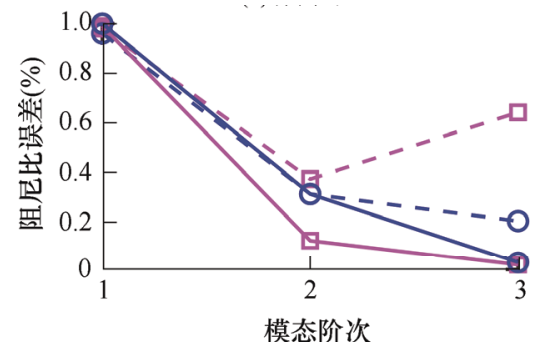

(b) 阻尼比误差

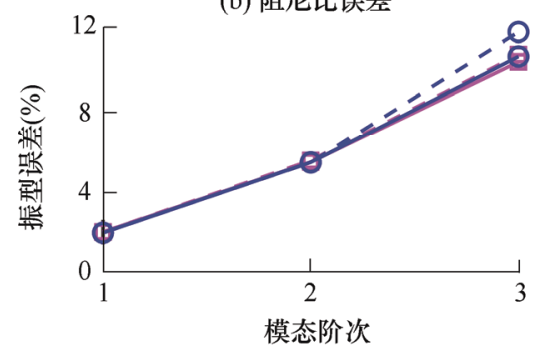

(c) 振型误差

图 11 识别结果与试验模态分析结果的误差

\section{5 结论}

（1）建立了基于传递率测量的伪频响函数矩阵 法。该方法首先构造多参考测点、多参考工况的伪 频响函数矩阵, 然后基于伪频响函数矩阵求解模态 频率和阻尼比、基于传递率函数矩阵求解模态振型, 最后建立相应的稳态图并结合以多参考测点平均的 传递率函数矩阵第 2 阶奇异值的倒数为基础的模态 指示函数来确定模态阶数与最终的模态结果。

(2) 仿真和试验案例结果均表明, 提出方法通 过充分利用所有响应数据有效缓解了已有伪频响函 数法识别结果稳定性不足的问题、能一定程度提高 模态识别精度, 且抗测量噪声干扰能力更优、更利 于自动化, 亦具有强 “谐波模态抑制” 能力。

(3) 在实际运用中, 提出方法需要的多种载 荷工况可通过改变被测对象的运转速度和负载等 实现。

\section{参 考 文 献}

[1] 刘宇飞, 辛克贵, 㚞健生, 等. 环境激励下结构模态参 数识别方法综述 [J]. 工程力学, 2014, 31(4): 46-53.

LIU Yufei, XIN Kegui, FAN Jiansheng, et al. A review of structure modal identification methods through ambient excitation[J]. Engineering Mechanics, 2014, 31(4): 46-53.

[2] 孙倩, 颜王吉, 任伟新. 基于响应传递比的桥梁结构工 作模态参数识别 [J]. 工程力学, 2017, 34(11): 194-201. SUN Qian, YAN Wangji, REN Weixin. Operational modal analysis for bridge engineering based on the dynamic transmissibility measurements[J].

Engineering
Mechanics, 2017, 34(11): 194-201.

[3] 张永祥, 刘心, 褚志刚, 等. 基于随机子空间法的模态 参数自动提取[J]. 机械工程学报, 2018, 54(9): 187-194. ZHANG Yongxiang, LIU Xin, CHU Zhigang, et al. Autonomous modal parameter extraction based on stochastic subspace identification[J]. Journal of Mechanical Engineering, 2018, 54(9): 187-194.

[4] MOHANTY P, RIXEN D J. Operational modal analysis in the presence of harmonic excitation[J]. Journal of Sound and Vibration, 2004, 270(1-2): 93-109.

[5] MAAMAR A, ABDELGHANI M, LE T P, et al. Operational modal identification in the presence of harmonic excitation[J]. Applied Acoustics, 2019, 147: 64-71.

[6] CHEN W, JIN M, HUANG J, et al. A method to distinguish harmonic frequencies and remove the harmonic effect in operational modal analysis of rotating structures[J]. Mechanical Systems and Signal Processing, 2021, 161: 107928.

[7] MODAK S V. Influence of a harmonic in the response on randomdec signature[J]. Mechanical Systems and Signal Processing, 2011, 25(7): 2673-2682.

[8] JACOBSEN N J. Identifying harmonic components in operational modal analysis[C]// Proceedings of the 12th International Congress on Sound and Vibration, July 11-14, 2005, Lisbon, Portugal. 2936-2943.

[9] 张义民, 张守元, 李鹤, 等. 运行模态分析中谐波模态 识别方法研究及应用 $[\mathrm{J}]$. 振动、测试与诊断, 2008, 28(3): 197-200, 298.

ZHANG Yimin, ZHANG Shouyuan, LI He, et al. Research on differentiation method of inherent mode and harmonic mode in operational modal analysis[J]. Journal of Vibration, Measurement and Diagnosis，2008，28(3): 197-200, 298.

[10] MOTTE K, WEIJTJENS W, DEVRIENDT C, et al. Operational modal analysis in the presence of harmonic excitations: A review[C]// Proceedings of the 33rd Conference and Exposition on Balancing Simulation and Testing, February 2-5, 2015, Orlando, Florida, USA. 379-395.

[11] 夏遵平, 王峡. 基于谱峭度的谐波模态检测方法 [J]. 工 程力学, 2013, 30(12): 255-258, 266.

XIA Zunping, WANG Tong. Detection of harmonic modes with spectral kurtosis[J]. Engineering Mechanics, 2013, 30(12): 255-258, 266.

[12] DEVRIENDT C, GUILLAUME P. Identification of modal parameters from transmissibility measurements[J]. Journal of Sound and Vibration, 2008, 314(1-2): 343-356.

[13] DEVRIENDT C, DE SITTER G, VANLANDUIT S, et 
al. Operational modal analysis in the presence of harmonic excitations by the use of transmissibility measurements[J]. Mechanical Systems and Signal Processing, 2009, 23(3): 621-635.

[14] PINTELON R, GUILLAUME P, ROLAIN Y, et al. Parametric identification of transfer functions in the frequency domain: A survey[J]. IEEE Transactions on Automatic Control, 1994, 39(11): 2245-2260.

[15] PEETERS B, VAN DER AUWERAER H, GUILLAUME $\mathrm{P}$, et al. The PolyMAX frequency-domain method: A new standard for modal parameter estimation?[J]. Shock and Vibration, 2004, 11(3-4): 395-409.

[16] DEVRIENDT C, WEIJTJENS W, DE SITTER G, et al. Combining multiple single-reference transmissibility functions in a unique matrix formulation for operational modal analysis[J]. Mechanical Systems and Signal Processing, 2013, 40(1): 278-287.

[17] DEVRIENDT C, DE SITTER G, GUILLAUME P. An operational modal analysis approach based on parametrically identified multivariable transmissibilities[J]. Mechanical Systems and Signal Processing, 2010, 24(5): 1250-1259.

[18] ARAUJO I G, LAIER J E. Operational modal analysis using SVD of power spectral density transmissibility matrices[J]. Mechanical Systems and Signal Processing, 2014, 46(1): 129-145.

[19] SCIONTI M, LANSLOTS J P. Stabilization diagrams: Pole identification using fuzzy clustering techniques[J]. Advances in Engineering Software, 2005, 36(11-12): 768-779.

[20] DE SITTER G, DEVRIENDT C, GUILLAUME P. Transmissibility-based operational modal analysis: Enhanced stabilisation diagrams[J]. Shock and Vibration, 2012, 19(5): 1085-1097.

[21] 禇志刚, 夏金风, 王光建, 等. 循环对称结构重根模态 振型相关性修正 [J]. 机械工程学报, 2014, 50(23): 59-65. CHU Zhigang, XIA Jinfeng, WANG Guangjian, et al. Correlation correction for doublet mode shapes of cyclic symmetric structures[J]. Journal of Mechanical Engineering, 2014, 50(23): 59-65.

作者简介: 陈涛, 男, 1995 年出生。主要研究方向为结构模态分析技术 理论及应用。

E-mail: ct0823@cqu.edu.cn

褚志刚(通信作者), 男, 1978 年出生, 博士, 教授, 博士研究生导师。 主要研究方向为振动噪声测量分析技术、声源识别技术理论及其应用。

E-mail: zgchu@cqu.edu.cn 